\title{
The optimal pursuit problem reduced to an infinite system of differential equations
}

\begin{abstract}
The optimal game problem reduced to an infinite system of differential equations with integral constraints on the players' controls is considered. The goal of the pursuer is to bring the system into the zeroth state, while the evader strives to prevent this. It is shown that Krasovskii's alternative is realized: the space of states is divided into two parts so that if the initial state lies in one part, completion of the pursuit is possible, and if it lies in the other part, evasion is possible. Constructive schemes for devising the optimal strategies of the players are proposed, and an explicit formula for the optimal pursuit time is derived.
\end{abstract}

Keyword: Explicit formula; Game problem; Infinite system of differential equations; Initial state; Integral constraints; One parts; Optimal strategies; Pursuit problems 